ARQGA / 982

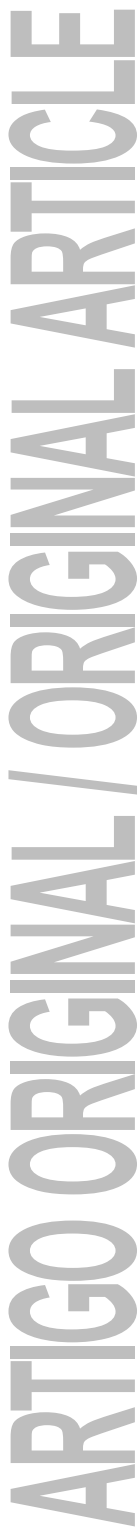

\title{
RETORNO AO TRABALHO DE PACIENTES ADULTOS SUBMETIDOS A TRANSPLANTE DE FÍGADO
}

\author{
Mônica Beatriz PAROLIN ${ }^{1}$, Júlio Cézar Uili COELHO ${ }^{2}$, \\ Patrícia Burda COSTA ${ }^{3}$, Silvânia Klug PIMENTEL ${ }^{3}$, \\ Lauro Estefano dos SANTOS-NETO ${ }^{4}$ e Stela Adami VAYEGO ${ }^{5}$
}

RESUMO - Racional - O transplante hepático é hoje o tratamento de eleição das doenças hepáticas avançadas. A melhoria da qualidade de vida após o transplante hepático tem sido objeto de inúmeros estudos e o restabelecimento da capacidade laborativa do individuo é um de seus indicadores. Objetivo - Avaliar o índice de retorno ao trabalho em adultos submetidos a transplante hepático e as causas para inatividade no pós-transplante. Pacientes e Métodos - Quarenta e um pacientes adultos (idade $\geq 18$ anos) submetidos a transplante hepático, entre setembro de 1991 a junho de 1999, com sobrevida póstransplante $\geq 9$ meses, boa função do enxerto e seguimento clínico regular foram avaliados em relação ao grau de inatividade no periodo pré e pós-transplante mediante aplicação de questionário. Resultados - Vinte e oito dos 41 pacientes transplantados (68\%) retornaram ao trabalho após o transplante hepático; o tempo médio decorrido entre a cirurgia do transplante e o retorno ao trabalho foi de 5,9 meses. Onze dos 28 pacientes ativos após o transplante (39\%) contribuiam efetivamente para a renda familiar. As causas de inatividade após o transplante hepático em 13 pacientes foram: aposentadoria precoce $(n=5)$, desemprego $(n=4)$ e incapacidade física $(n=4)$. Conclusão - O transplante hepático bem sucedido permite a um número considerável de pacientes retornar à atividade laborativa, reassumindo suas funções sociais.

DESCRITORES - Transplante de fígado. Hepatopatias. Qualidade de vida. Atividades cotidianas.

\section{INTRODUÇÃO}

Durante a última década os expressivos resultados nas curvas de sobrevida obtidos nos programas de transplante hepático (TH) firmaram essa modalidade terapêutica como tratamento de eleição nas doenças hepáticas avançadas.

A melhoria da qualidade de vida no período póstransplante tem sido alvo de inúmeros estudos, pois deve ser um dos objetivos desse recurso terapêutico ${ }^{(3,4,6,7,8,9)}$. O restabelecimento da capacidade laborativa do indivíduo após o TH é um dos aspectos que refletem seu impacto positivo na qualidade de vida do receptor. Além disso, o TH demanda considerável investimento técnico e financeiro e a reintegração do indivíduo transplantado as suas funções sociais é uma forma de retribuir à sociedade tal investimento.

O objetivo do presente estudo é determinar em nosso meio o índice de retorno ao trabalho de adultos submetidos a TH que apresentam boa função do enxerto e identificar as causas para inatividade no período pós-transplante.

\footnotetext{
Serviço de Transplante Hepático do Hospital de Clínicas da Universidade Federal do Paraná (UFPR) e Departamento de Estatística da UFPR.

Médica Hepatologista do Serviço de Transplante Hepático do HC-UFPR.

Professor Titular e Chefe da Disciplina de Cirurgia do Aparelho Digestivo e do Serviço de Transplante Hepático do HC-UFPR.

Médica Residente do Serviço de Cirurgia do Aparelho Digestivo do HC-UFPR.

Acadêmica do Curso de Medicina da UFPR.

Professora Assistente do Departamento de Estatística da UFPR.
}

Endereço para correspondência: Dra. Mônica Beatriz Parolin - Rua Lamenha Lins, 2280 - 80220-080 - Curitiba, PR. e-mail: mbparolin@hotmail.com 


\section{PACIENTES E MÉTODOS}

No período de setembro de 1991 a junho de 1999 foram realizados 107 transplantes ortotópicos de fígado em 101 adultos (idade $\geq 18$ anos) no Serviço de Transplante Hepático do Hospital de Clínicas da Universidade Federal do Paraná, Curitiba, PR. No período de maio a julho de 1999 os arquivos médicos do referido Serviço foram revisados para identificar pacientes que preenchessem os seguintes critérios de inclusão: sobrevida após TH $\geq 9$ meses, boa função do enxerto, acompanhamento clínico regular e sem antecedentes de aposentadoria por tempo de serviço precedendo o surgimento dos sintomas da doença hepática.

Os pacientes que preenchiam os critérios de inclusão para o estudo foram avaliados pela resposta a questionário aplicado por ocasião das visitas médicas ou por telefone, quando se encontravam distantes do centro. Os seguintes itens foram pesquisados: grau de escolaridade $\left(1^{\circ}, 2^{\circ}\right.$ e $3^{\circ}$ grau, completo ou incompleto), tipo de atividade no prétransplante, grau e duração da inatividade na fase pré-transplante (parcial, total ou nula), duração da inatividade no pós-transplante, estado atual de atividade (ativo ou inativo), contribuição para renda familiar. Os dados clínicos coletados dos prontuários médicos incluíram: idade, sexo, etiologia da doença hepática, tempo de sobrevida após o transplante, condição clínica na época do transplante, de acordo com a classificação de Child-Pugh modificada ${ }^{(5)}$.

Quarenta e um pacientes submetidos a TH preencheram os critérios de inclusão e constituíram a amostra estudada. $\mathrm{O}$ grupo consistiu de 24 homens e 17 mulheres, com idade média de 44,2 \pm 13,8 anos (19-67 anos) e tempo médio de sobrevida pós-TH de 32,3 $\pm 16,9$ meses (9-71 meses) e cujas principais características clínicas e bioquímicas estão apresentadas na Tabela 1. A principal etiologia da doença hepática no grupo estudado foi cirrose hepática devido a infecção crônica pelo vírus da hepatite $\mathrm{C}(\mathrm{n}=11,26,8 \%)$. Em um dos 11 casos de cirrose pelo vírus da hepatite $\mathrm{C}$ havia a presença concomitante de hepatocarcinoma restrito ao fígado. A infecção pelo vírus da hepatite $\mathrm{C}$ esteve presente como co-fator de injúria hepática em dois dos cinco pacientes com doença hepática alcoólica. Trinta e sete dos 41 (90\%) pacientes avaliados apresentavam na ocasião do transplante, disfunção hepática moderada ou grave, de acordo com a classificação de Child-Pugh modificada (Tabela 1). Os quatro indivíduos com boa reserva funcional hepática (Child A) tinham como indicação para transplante, respectivamente: cirrose hepática pelo vírus da hepatite $\mathrm{C}$ complicada com hepatocarcinoma, adenoma gigante de fígado, polineuropatia amiloidótica familial e doença de Wilson com manifestações neurológicas refratárias ao tratamento clínico.

Os 41 pacientes transplantados apresentavam os seguintes graus de escolaridade: 10 completaram o $1^{\circ}$ grau $(24,4 \%), 11$ o $2^{\circ}$ grau $(26,8 \%)$ e 8 o $3^{\circ}$ grau (19,5\%); apenas 12 dos $41(29,3 \%)$ não tinham o $1^{\circ}$ grau completo. Em relação ao tipo de atividade laborativa exercida pelos pacientes na fase pré-transplante, com exceção de 2 agricultores, os 39 demais (95\%) desempenhavam funções que não exigiam esforço físico intenso.

TABELA 1 - Características dos 41 pacientes adultos submetidos a $\mathrm{TH}$

\begin{tabular}{lc} 
Características & \\
\hline Sexo (masculino/feminino) & $24 / 17$ \\
Idade em anos (média \pm desvio padrão) & $44,2 \pm 13,8$ \\
Classificação de Child-Pugh modificada (n) & \\
Classe A & 4 \\
Classe B & 21 \\
Classe C & 16 \\
Etiologia da doença hepática (n) & \\
Hepatite C* & 11 \\
Doença colestática crônica & 09 \\
Autoimune & 06 \\
Criptogênica & 05 \\
Álcool & 05 \\
Miscelânea** & 05
\end{tabular}

* Presença de hepatocarcinoma restrito ao fígado em um dos pacientes com cirrose por hepatite $\mathrm{C}$

** Miscelânea: adenoma gigante de fígado $(\mathrm{n}=1)$, polineuropatia amiloidótica familial $(\mathrm{n}=1)$, doença de Wilson $(\mathrm{n}=1)$, síndrome de Budd-Chiari $(\mathrm{n}=1)$, hemocromatose hereditária $(\mathrm{n}=1)$

Trinta e um pacientes $(75,6 \%)$ necessitaram de afastamento do trabalho na fase pré-transplante devido ao comprometimento importante do estado de saúde pela doença hepática. Onze dos 31 pacientes $(35,4 \%)$ apresentavam inatividade total e $16(51,6 \%)$ parcial. A mediana do período de inatividade na fase pré-transplante foi de 16 meses, variando de 1 a 96 meses.

Os resultados obtidos foram expressos em média e desvio padrão (variáveis contínuas) ou percentagens. Na comparação das médias das variáveis idade na ocasião do transplante e tempo de inatividade pré-transplante foi empregado o teste $t$ de Student, ao nível de significância de 5\%. Para as variáveis classe de Child-Pugh e condição de atividade pré e pós-transplante (ativo/inativo) foram usados os testes não-paramétricos do Qui-quadrado ou o teste exato de Fisher, ao nível de significância de 5\% (Statistica for Windows, versão 5.1, 1997, Stat Soft, Inc).

\section{RESULTADOS}

Após o TH, 28 dos 41 pacientes entrevistados (68,3\%) retornaram às atividades laborativas. $\mathrm{O}$ tempo médio de retorno ao trabalho após o transplante foi de 5,9 $\pm 3,4$ meses, variando de 2 a 12 meses. Seis donas de casa e dois estudantes que reassumiram suas atividades após 
o TH foram considerados ativos. Onze dos 28 pacientes (39,3\%) que retornaram ao trabalho após o transplante contribuíam ativamente para a renda familiar.

O tempo médio de inatividade no período pré-transplante foi significativamente menor nos pacientes que retornaram às atividades laborativas quando comparado aos pacientes que permaneceram inativos $(15,0 \pm 10,0$ meses vs. $64 \pm 30,2$ meses, $P<0,001)$.

A idade do receptor também mostrou ter influência no retorno ao trabalho após o transplante. A idade média desses pacientes foi significativamente menor que a idade dos que permaneciam inativos até a época em que o presente estudo foi realizado $(37,1 \pm 10,5$ anos vs. 59,5 $\pm 4,1$ anos, $P<0,001)$.

Não se observou diferença estatisticamente significativa quanto ao grau de reintegração ao trabalho, em relação à condição clínica do paciente (classificação de Child-Pugh) na ocasião do TH: 2 dos 4 pacientes Child A, 14 dos 21 Child B e 12 dos 16 Child C retornaram ao trabalho $(P=0,61)$.

As causas de não retorno ao trabalho após o TH nos 13 pacientes que permaneceram inativos foram: aposentadoria precoce $(n=5)$, desemprego $(n=4)$ e incapacidade física $(n=4)$. Nove dos 13 pacientes que não retornaram à atividade remunerada, declararam-se, em análise subjetiva, capazes de retornar ao trabalho por apresentarem boas condições de saúde.

\section{DISCUSSÃO}

$\mathrm{O}$ TH é hoje procedimento fundamental no arsenal terapêutico das doenças hepáticas avançadas e que exige grande investimento financeiro e técnico, além da mobilização da família e da sociedade. Inúmeros estudos demonstram que essa modalidade terapêutica proporciona melhora considerável da qualidade de vida em grande número de transplantados ${ }^{(3,4,6,7,8,9)}$. A possibilidade do $\mathrm{TH}$ permitir o retorno do paciente a sua atividade laborativa é um dos indicadores do sucesso desse tratamento.

O presente estudo documentou que o TH bem sucedido permitiu o retorno ao trabalho em $68 \%$ dos receptores adultos com enxerto funcionante. $\mathrm{O}$ valor social do TH é também demonstrado pela contribuição efetiva de $39 \%$ dos receptores ativos na renda familiar. Pelo fato das doenças hepáticas avançadas de diferentes causas afetarem, em sua maioria, pacientes na fase mais produtiva de suas vidas, a reintegração ao trabalho é importante parâmetro quando se analisa o impacto positivo do TH na qualidade de vida dos receptores.

Relatos oriundos de serviços de $\mathrm{TH}$ em outros países têm identificado índices de retorno ao trabalho após o transplante que variam de $27 \%$ a $69 \%^{(1,2,6,7)}$. Em publicação recente sob a forma de resumo $^{(2)}$, um Serviço brasileiro documenta retorno ao trabalho em 9 de 17 (43\%) receptores de TH. A comparação dos valores encontrados em diferentes centros é difícil pois a maioria dos estudos é retrospectiva, não controla fatores como tempo pós-transplante e apresenta fatores de exclusão que impossibilitam a generalização dos resultados.

No presente estudo, 13 dos 41 (32\%) receptores de TH entrevistados não retornaram ao trabalho remunerado. Nove dos 13 pacientes revelaram-se, em análise subjetiva, como capazes para o trabalho por terem condição melhor do seu estado de saúde e apontaram como causa para a inatividade a aposentadoria precoce $(\mathrm{n}=5)$ e a falta de oportunidade no mercado de trabalho $(\mathrm{n}=4)$. Portanto, número considerável de receptores de TH apresenta melhora substancial na qualidade de vida, sem no entanto, retornar à atividade laborativa.

Diferentes fatores influenciam na reintegração do indivíduo ao trabalho após o TH. ADAMS et al. ${ }^{(1)}$, avaliando 203 pacientes adultos submetidos a TH e sobrevida pós-transplante $\geq 9$ meses, encontraram índice de retorno ao trabalho de $57 \%$. Identificaram fatores que estavam associados ao retorno desses pacientes ao trabalho no período pós-transplante: duração da inatividade antes do TH (apenas $33,6 \%$ dos inativos por mais de 5 anos retornaram ao trabalho após o transplante), idade do paciente e tipo de ocupação antes de tornarse inativo. $\mathrm{O}$ fato de receptores mais idosos retornarem com menor freqüência ao trabalho não surpreende, visto que procuram aposentadoria mais precocemente e sua habilidade em encontrar emprego decresce com a idade. Em relação ao tipo de trabalho exercido no pré-transplante, receptores cujo emprego consistia em tarefas que exigiam maior esforço físico, foram menos propensos a retornar ao trabalho após o TH, quando comparados aos que desenvolviam atividades mais intelectuais. Tal dificuldade poderia, segundo ADAMS et al. ${ }^{(1)}$, estar relacionada ao menor nível de escolaridade daqueles candidatos, o que dificultaria seu retorno ao mercado de trabalho. À semelhança dos dados encontrados por ADAMS et al. ${ }^{(1)}$, o presente estudo também detectou a influência da idade do receptor e do tempo de inatividade na fase pré-transplante com a reintegração ao trabalho. A idade média e o tempo médio de inatividade pré-tansplante dos receptores que retornaram ao trabalho foram significativamente menor, quando comparados aos que permaneceram inativos $(37,1 \pm 10,5$ anos vs. $59,5 \pm 4,1$ anos, $P<0,001$ e $15,0 \pm 10,0$ meses vs. $64 \pm 30,2$ meses, $P<0,001$, respectivamente). LEVY et al.(7) demonstraram que a reintegração do indivíduo ao trabalho ocorre gradualmente e tende a melhorar ao longo dos anos. Estudando 360 pacientes submetidos a TH, esses autores demonstraram que $39 \%$ dos indivíduos estavam trabalhando após o primeiro ano de TH; o nível de emprego melhorou no segundo ano para $57,5 \%$ e atingiu $70 \%$ no quinto ano de seguimento.

O presente estudo não identificou correlação entre a condição clínica do paciente na ocasião do transplante (de acordo com a classificação de Child-Pugh) e o índice de retorno ao trabalho. ADAMS et al. ${ }^{(1)}$ não observaram diferença estatisticamente significativa entre 
os pacientes ativos e inativos após o TH em relação a variáveis prétransplante como sexo, etiologia da doença hepática e condição clínica do paciente na época do transplante. Cabe ressaltar que o desenho do estudo desenvolvido por esses autores, e que serviu como referência para o presente estudo, excluiu pacientes com sobrevida póstransplante inferior a 9 meses. Assim, a amostra selecionada não reflete o universo total de pacientes submetidos a $\mathrm{TH}$, e pode comprometer a análise do real impacto da condição clínica na ocasião do transplante na reabilitação do indivíduo.
O presente estudo demonstra que o TH bem sucedido permite que um número considerável de pacientes se reintegre às atividades profissionais. Tornar o paciente apto para o trabalho deve ser visto como um dos objetivos do TH, considerando-se que as doenças hepáticas tendem a afetar os indivíduos na fase mais produtiva das suas vidas. $\mathrm{O}$ melhor conhecimento das dificuldade que os pacientes encontram na fase de reintegração às atividades laborativas após o $\mathrm{TH}$, poderá auxiliar as equipes de transplante a elaborar programas de apoio social e psicológico que os ajude a alcançar a reabilitação funcional plena.

Parolin MB, Coelho JCU, Costa PB, Pimentel SK, Santos-Neto LE, Vayego SA. Return to work of adults after liver transplantation. Arq Gastroenterol 2001;38(3):172-175.

ABSTRACT - Background - Orthotopic liver transplantation is an established therapy for patients with end-stage liver disease. In the last years more attention has been given for the improvement of the quality of life after liver transplantation, and the return to important life pursuits, such as employment, are important goals of this type of therapy. Aim - To assess the work's return rate in liver transplant recipients and the reasons for inability after liver transplant. Patients and Methods - Forty-one adults (age $\geq 18$ years) who underwent liver transplantation at our institution, between September 1991 and June 1999, with a post-transplant survival $\geq 9$ months, good graft function and a regular clinical follow-up were enrolled for the study. A questionnaire that measured aspects of inactivity before and after liver transplantation had been applied. Results - Thirty-one of 41 patients were unable to work due end-stage liver disease before liver transplantation. Return to work was observed in 28 of 41 patients (68\%) in a mean time of 5,9 months after the surgical procedure. Eleven of 28 active patients after liver transplant (39\%) contribute significantly to family income. The reasons for inactivity after liver transplantation were: early retirement $(n=5)$, unemployment $(n=4)$, and physical disability $(n=4)$. Conclusion - Successful liver transplantation not only improves the survival rate but allows patients to return to social function and workforce.

HEADINGS - Liver transplantation. Liver diseases. Quality of life. Activities of daily living.

\section{REFERÊNCIAS BIBLIOGRÁFICAS}

1. Adams PC, Ghent CN, Grant DR, Wall WJ. Employment after liver transplantation Hepatology 1995;21:140-4.

2. Alvares da Silva MR, Sommer JW, Laux M, Waechter FL, Sampaio JA, Pinto RD, Francisconi CF, Pereira Lima LM. Assessing quality-of-life before and following liver transplantation. Hepatology 1999;30:663A.

3. Bonsel GJ, Essink-Bot L, Klompmaker IJ, Slooff MJH. Assessment of the quality of life before and following liver transplantation. Transplantation 1992;53:796800 .

4. Bravata DM, Olkin I, Barnato AE, Keeffe EB, Owens DK. Health-related quality of life after liver transplantation: a meta-analysis. Liver Transplant Surg 1999;5:318-31.

5. Child CG, Turcotte JG. Major problems in clinical surgery. In: Child CG, editor. The liver and portal hypertension. New York: WB Saunders; 1964.
6. Gross CR, Malinchoc M, Kim WR, Evans RW, Wiesner RH, Petz JL, Crippin JS, Klintmalm GB, Levy MF, Ricci P, Therneau TM, Dickson R. Quality of life before and after liver transplantation for cholestatic liver disease. Hepatology 1999 29:356-64.

7. Levy MF, Jennings L, Abouljoud MS, Mulligan DC, Goldstein RM, Husberg BS, Gonwa TA, Klintmalm GB. Quality of life improvements at one, two, and five years after liver transplantation. Transplantation 1995;59:515-8.

8. Price CE, Lowe D, Cohen AT, Reid FDA, Forbes GM, McEwen J, Williams R. Prospective study of the quality of life in patients assessed for liver transplantation: outcome in transplanted and not transplanted groups. J R Soc Med 1995;88:130-5.

9. Tarter RE, Switala J, Arria A, Plail J, Van Thiel D. Quality of life before and after orthotopic hepatic transplantation. Arch Intern Med 1991;152:1521-6.

Recebido em 1/2/2001. Aprovado em 7/8/2001 